Title of the dossier/Titre du dossier

\title{
Afterglows after Swift
}

\author{
O. Godet ${ }^{\mathrm{a}}$, R. Mochkovitch ${ }^{\mathrm{b}}$

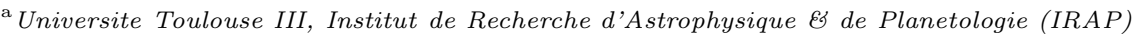 \\ 9 Av. du Colonel Roche, 31028 Toulouse Cedex 4, France \\ ${ }^{\mathrm{b}}$ Institut d'Astrophysique de Paris, UMR 7095, CNRS - Université Pierre et Marie Curie \\ 98bis Bd Arago, 75014 Paris, France
}

\begin{abstract}
Since their discovery by the Beppo-SAX satellite in 1997, gamma-ray burst afterglows have attracted an evergrowing interest. They have allowed redshift measurements that have confirmed that gamma-ray bursts are located at cosmological distances. Their study covers a huge range both in time (from one minute to several months after the trigger) and energy (from the $\mathrm{GeV}$ to radio domains). The purpose of this review is first to give a short historical account of afterglow research and describe the main observational results with a special attention to the early afterglow revealed by Swift. We then present the standard afterglow model as it has been developed in the pre-Swift era and show how it is challenged by the recent Swift and Fermi results. We finally discuss different options (within the standard framework or implying a change of paradigm) that have been proposed to solve the current problems.
\end{abstract}

To cite this article: O. Godet, R. Mochkovitch, C. R. Physique 12 (2011).

\section{Résumé}

Les rémanences après Swift. Depuis leur découverte par le satelite Beppo-SAX en 1997, les rémanences des sursauts gamma ont suscité un intérêt sans cesse croissant. Elles ont permis les mesures de redshifts qui ont confirmé que les sursauts sont situés à distance cosmologique. Leur étude couvre un vaste domaine aussi bien temporel (de une minute à plusieurs mois après le sursaut) que spectral (depuis les GeV jusqu'à la radio). Le but de cette revue est d'abord de faire un court rappel historique de la recherche sur les rémanences puis de décrire les principaux résultats observationnels avec une attention particulière pour la rémanence précoce, révélée par Swift. Nous présentons ensuite le modèle standard tel qu'il fut développé durant l'ère pré-Swift et montrons comment il est mis en défaut par les résultats récents de Swift et Fermi. Nous discutons enfin les propositions (dans le cadre standard ou impliquant au contraire un changement de paradigme) qui ont été avancées pour résoudre les différents problèmes.

Pour citer cet article : O.Godet, R. Mochkovitch, C. R. Physique 12 (2011).

Key words: Gamma-Ray Bursts; Afterglow; Hydrodynamics; Radiative Processes ; Multiwavelength Observations

Mots-clés : Sursauts Gamma; Rémanence; Hydrodynamique; Processus Radiatifs; Observations Multi-longueurs d'onde

Email addresses: olivier.godet@cesr.fr (O. Godet), mochko@iap.fr (R. Mochkovitch). 


\section{Introduction}

Even before the discovery of the first afterglow by Beppo-SAX it was suspected that the prompt phase of gamma-ray bursts (hereafter GRBs) should be followed by some long lasting emissions at longer wavelengths. By analogy with supernova remnants it seemed natural to believe that a fraction of the energy dissipated when the relativistic ejecta is decelerated by the circumburst medium would be radiated, giving rise to an "afterglow" .

The detection of the first X-ray afterglow associated with GRB 970228 on February 28th, 1997 marked a milestone in the GRB study [1]. The 3 arcminute X-ray error box was searched with the William Herschel and Isaac Newton telescopes at La Palma and a weak optical counterpart was discovered. On March $1^{\text {st }}$ it had a visual magnitude $V=21.3$ and declined below 24 after a week. A faint galaxy $(R=24)$ was found within 0.2 arcsecond from the counterpart. It was the likely host of the burst and this, for the first time, provided a direct evidence that GRBs were located at very large (cosmological) distances. Three months later another burst (GRB 970508) was localized by Beppo-SAX and a spectrum of the afterglow taken by the Keck telescope showed redshifted lines of silicon and iron at $z=0.77$ and $z=0.835$ (the largest redshift being that of the host galaxy and the smaller one corresponding to an intervening absorption system). This first redshift definitely confirmed the cosmological distance scale. In December of the same year GRB 971214 reached $z=3.42$.

From that moment, it became possible to identify long-GRB host galaxies leading in turn to constraints on GRB progenitors, to have access to physical quantities that enabled to intensively test theoretical models. This outstanding achievement was made possible thanks to the Italian-Dutch BeppoSAX (e.g. [2]) mission with the spacecraft being able to slew within a few hours of an alert to bring the GRB trigger position in the field of view of the narrow field X-ray instruments (NFIs) that could further refine the error box. This allowed, for the first time, ground facilities to observe quickly the GRB multi-wavelength afterglow. HETE 2 [3], launched in 2000, had similar capabilities and the two satellites collected a total of nearly 40 redshifts from 1997 to 2005.

Another milestone in GRB study was marked by the launch of the Swift mission [4] in November 2004 that brings the BeppoSAX and HETE 2 observing strategy to the best refinement by associating a slew capability of a few tens of seconds with a platform carrying a wide-field coded-mask telescope providing the trigger and two very sensitive NFIs in X-rays and optical. This led Swift: i) to fully characterise the emission, in particular in X-rays, from a temporal interval (between a few minutes and a few hours from the prompt emission) that was previously not accessible, the so-called early afterglow; ii) to discover the multi-wavelength afterglows of the prior-elusive

short GRBs, which led in turn to better understand their nature; iii) to detect several high-redshift GRBs of which 3 events with $z=6.3,6.7$ and 8.2.

GRB afterglows convey broadband information on the physics of the GRB phenomenon itself, but also on the GRB host galaxies and the GRB environment and hence on the GRB progenitors, and as cosmological probes they allow to study the evolution of baryons with redshift or even possibly the re-ionisation of the Universe for the furthest ones. Here, we discuss about our current state-of-the-art understanding of the afterglow physics in the Swift and Fermi era. The use of GRBs as cosmological tools as well as the nature of their progenitors is addressed respectively in [5] \& [6] (this issue). We focus on the behaviour of the afterglow in X-rays that was intensively characterised with Swift and the related physics, and then we discuss how the observations in other wavelengths fit or not in this framework.

The review is organised as follows: in Section 2, we describe the standard afterglow model. In Section 3, we briefly present the Swift mission and then we detail the afterglow X-ray canonical light curve revealed by $S w i f t$. We discuss in some length its physical interpretation as well as what observations in other wavelengths tell us. We discuss in Section 4 the afterglow properties of different classes of GRBs. In Section 5, we give our view on some of the outstanding current open issues that future missions and theoretical developments will need to address.

\section{Standard model of the afterglow}

Following the discovery of the first afterglows, models were readily constructed to interpret the new wealth of data. Any model of the afterglow consists in two parts: (i) an hydrodynamical description of the deceleration of the relativistic burst ejecta by the circumstellar medium and (ii) the identification of the radiative processes at work. The deceleration leads to the usual two-shock picture: a forward shock propagating in the external medium and a reverse shock sweeping back into the ejecta, the two shocked regions being separated by a contact discontinuity. The standard model assumes that the afterglow results from the synchrotron emission of electrons accelerated in the forward shock [7]. In the basic version of the model the hydrodynamics is described by the Blandford-McKee 
[8] solution (a relativistic analogue to the classical Sedov self-similar blast wave model). Behind the shock, the electrons are accelerated to a non-thermal power law distribution and then emit synchrotron radiation in the local magnetic field. The dynamics is fixed by the total explosion kinetic energy $E_{\mathrm{K}}$ (in $\mathrm{erg} \mathrm{sr}^{-1}$ since the ejecta is supposed to be beamed in two opposite jets of initial opening angle $\theta_{j}$ ) and the external density (a uniform or wind-like medium). The instantaneous synchrotron spectrum at a given shock location depends on three physical quantities: the slope $p$ and minimum Lorentz factor $\gamma_{m}$ of the initial distribution of electrons, and the magnetic field strength $B$. The last two are estimated by assuming that respective fractions $\epsilon_{e}$ and $\epsilon_{B}$ of the dissipated energy (in the local fluid frame) are used to accelerate electrons and amplify the magnetic field [9]. Comparing the hydrodynamical expansion time to the synchrotron cooling time then leads to define the Lorentz factor $\gamma_{c}$ : electrons with Lorentz factors $\gamma>\gamma_{c}$ can radiate efficiently while those with $\gamma<\gamma_{c}$ mostly loose their energy by adiabatic expansion. Hence, depending on the relative order of $\gamma_{m}$ and $\gamma_{c}$ one distinguishes two cooling regimes: fast cooling $\left(\gamma_{m}>\gamma_{c}\right)$ and slow cooling $\left(\gamma_{m}<\gamma_{c}\right)$. [10] obtained the expected spectra (for a power law distribution of injected electrons) in the two cases. Considering the synchrotron frequencies $\nu_{m}$ and $\nu_{c}$ respectively associated to $\gamma_{m}$ and $\gamma_{c}\left(\nu_{\text {syn }} \propto B \gamma^{2}\right)$ the elementary spectra are made of three power law segments for $\nu<\min \left(\nu_{m}\right.$, $\left.\nu_{c}\right), \min \left(\nu_{m}, \nu_{c}\right)<\nu<\max \left(\nu_{m}, \nu_{c}\right)$ and $\nu>\max \left(\nu_{m}, \nu_{c}\right)$. A fourth segment appears at low frequency when the possibility of self-absorption of the synchrotron radiation by the emitting electrons is taken into account. Coupling this result to the Blandford-McKee solution it is then possible to predict the afterglow light curve at a given frequency $\left(F_{\nu} \propto t^{-\alpha} \nu^{-\beta}\right)$ which is also made of several power law segments (with the so-called closure relations coupling the temporal and spectral indices $\alpha$ and $\beta$, see e.g. [11]). This was first done considering a forward shock propagating into a constant density interstellar medium (ISM)[10]. [12] and [13] then obtained similar solutions in a stellar wind environment. During the pre-Swift era this simple model appeared to give a fairly good account of the available observations (e.g. $[14,15,16])$. Multiwavelength fits of the data were used to constrain burst parameters such as the kinetic energy, external density or $\epsilon_{e}, \epsilon_{B}$ and $p$. Surprisingly, a uniform external medium appeared to be preferred in most cases while the progenitors of long bursts (the only ones with detailed afterglow observations in the pre-Swift era) are supposed to be Wolf-Rayet stars, which are normally thought to have strong winds. Another potential problem comes from the relatively high efficiency of the prompt emission mechanism implied by these results: the ratio $E_{\gamma} / E_{\mathrm{K}}$ between the energy measured in gamma-rays and the kinetic energy obtained from the fits is often larger than $10 \%$. Such an efficiency is difficult to reach by internal shocks, the most discussed mechanism for the prompt emission.

\section{The Swift era}

\subsection{The Swift mission}

The Swift mission [4] is a NASA, Italian and UK satellite launched in November 2004 to study GRBs with several challenging goals, amongst them: i) the detection of high-redshift GRBs; ii) the identification and characterisation of the afterglows from short GRBs; iii) the sampling of the afterglow emission in X-rays and optical from a few tens of seconds after the trigger to several days, thanks to rapid slew capability of about 1-2 minutes. To do so, Swift embarked three instruments: the Burst Alert Telescope (BAT [85]), a 15-150 keV and 2 sr coded-mask camera with a $5200 \mathrm{~cm}^{2}$ geometrical area in charge of the detection and primary localisation of GRBs with a typical accuracy of $3^{\prime}$ in radius at a $90 \%$ confidence level and two narrow-field instruments. The X-Ray Telescope (XRT [18]) and the UV/Optical Telescope (UVOT [19]) enable to refine the GRB error box after the spacecraft slew with typical accuracy in radius of better than $3^{\prime \prime}$ and $<1^{\prime \prime}$ at a $90 \%$ confidence level, respectively. The XRT is a $0.3-10 \mathrm{keV}$ Wolter-I telescope with a focal length of $3.5 \mathrm{~m}$, an effective area of $110 \mathrm{~cm}^{2}$ at $1.5 \mathrm{keV}$ and a field of view of $23.6 \times 23.6$ arcminutes. The UVOT is a modified Ritchey-Chrétien telescope with a field of view of 17 $\times 17$ arc-minute covering the $170-650 \mathrm{~nm}$ range.

Excluding the GRBs found by BATSE, 915 GRBs have been detected from 1997 to 2010. From this total, $65 \%, \sim 43 \%$ and $\sim 8 \%$ have been also seen in X-rays, IR/Optical and radio, respectively (see Fig. 1). From its launch to the end of the year 2010, Swift found 547 GRBs ( $\sim 60 \%$ of the 915 GRBs since 1997) of which more than $90 \%$ were also observed by the XRT and only $\sim 40 \%$ by the UVOT. It is interesting to note that even if ground follow-ups increase the fraction of GRBs detected in IR/Optical by $\sim 15-20 \%$, this fraction is still less than that observed in X-rays. The deficit is not the result of a lack of sensitivity of the UVOT and ground instruments, but more intrinsic to GRBs which appear for some of them as dark in IR/optical (e.g. [20,21] - cf. Section 3.3.1). As 


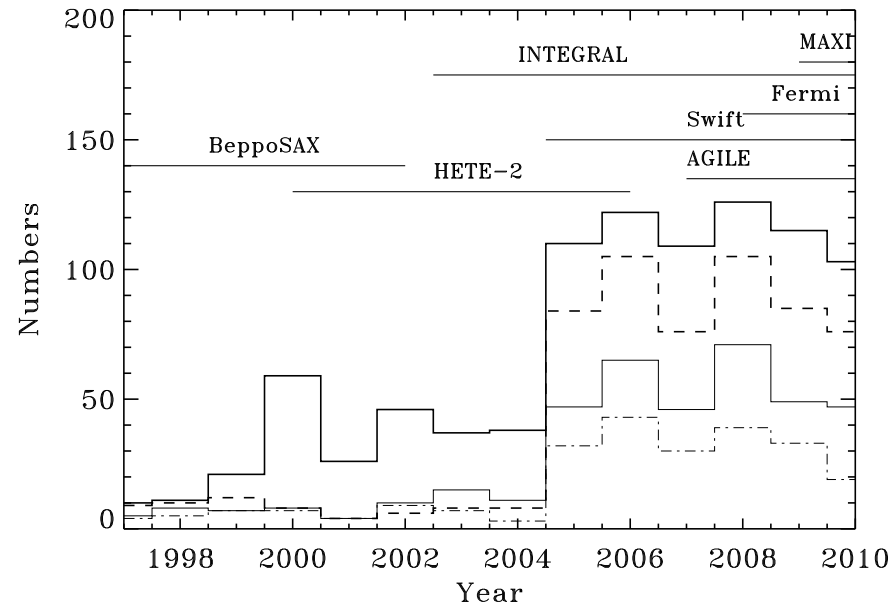

Figure 1. Plot showing the number of GRBs (thick solid line) detected by various gamma-ray instruments from 1997 to 2010 , the number of GRBs with detected X-ray (thick dashed line) and optical (thin solid line) afterglows, the number of GRBs with measured spectroscopic redshift (thin dotted-dashed line).

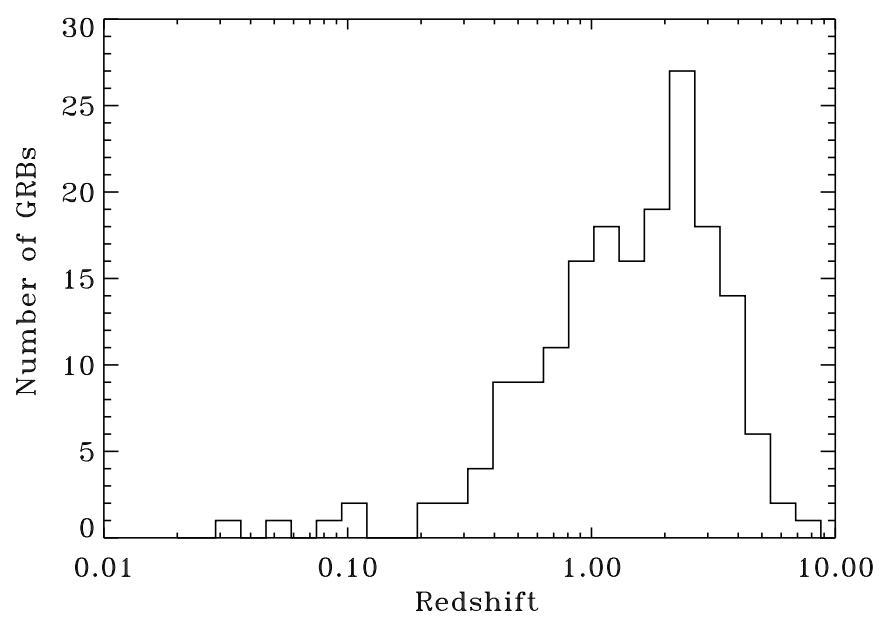

Figure 2. Distribution of measured spectroscopic redshifts up to the end of 2010 .

seen in Fig. 1 and 2, not only Swift brought a large sample of GRBs, it also increased by a factor of about 3 the number of GRBs with a measured spectroscopic redshift. The average redshift for Swift GRBs is around 2.0 (using spectrospic redshift measurements from 2005 to the end of 2010) against 1.2 prior to Swift (see e.g. [22]). This results from the higher sensitivity of the BAT that detects fainter GRBs when compared to previous GRB trigger cameras. Another tremendous achievement of Swift is its capability to follow the afterglow emission from a few tens of seconds (in some cases at a time when the prompt gamma-ray ray emission is still ongoing) to several days or couple of weeks and in some exceptional cases over more than a month as for GRB 060729 (see Fig. 4; $[23])$. 


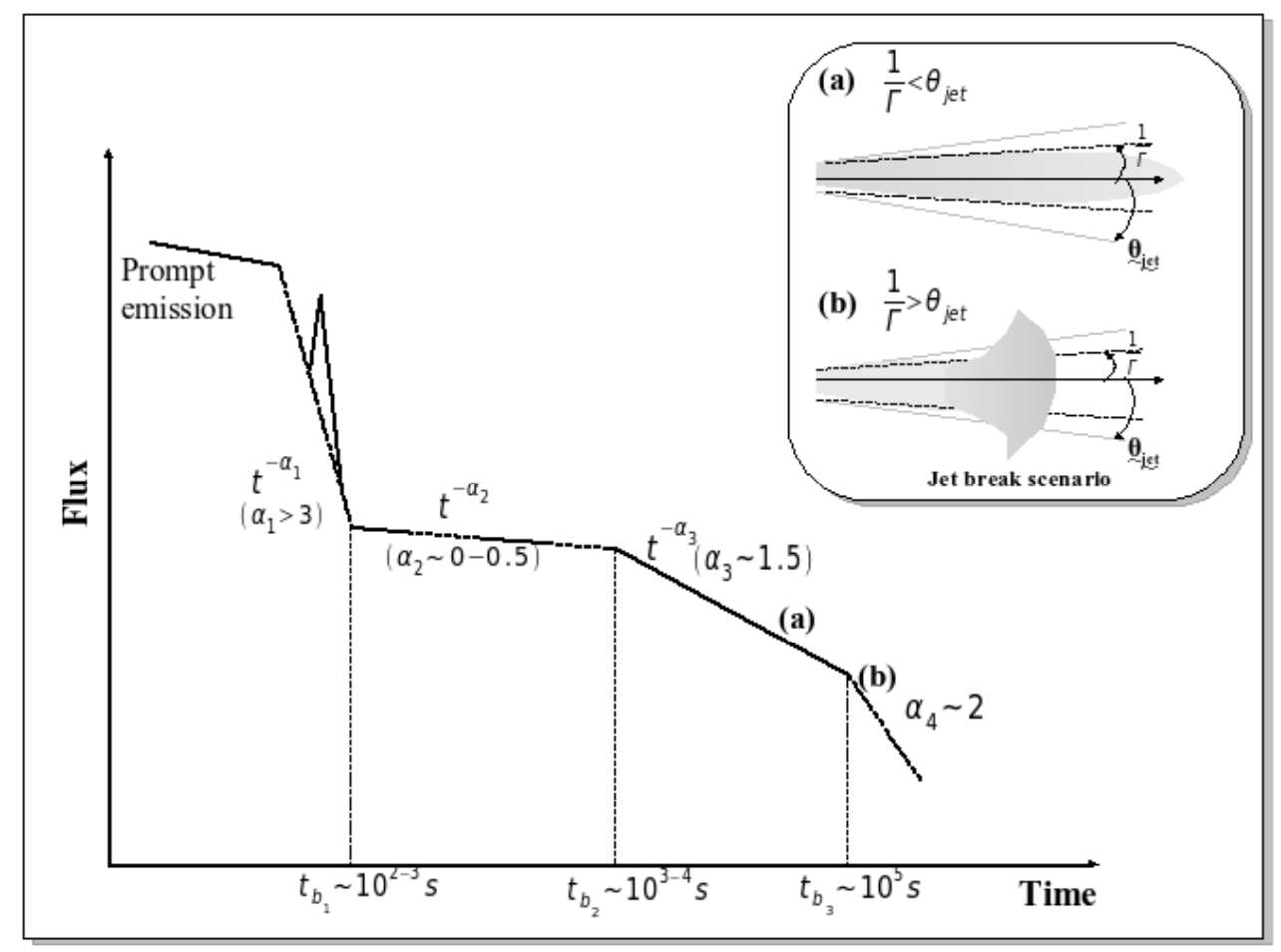

Figure 3. Scheme showing the X-ray canonical light-curve. The dotted segments correspond to temporal sequences that are not observed in every GRB light-curve.

\subsection{Swift X-ray observations}

\subsubsection{The canonical X-ray light curve}

From the data collected by the XRT a general pattern rapidly emerged concerning the shape of the early X-ray afterglow of long GRBs (see Fig. 3). Before the standard behavior $F_{X} \propto t^{-\alpha}$ with $\alpha=1-1.5$ which was known before Swift, the canonical light curve first shows a steep decay (with $\alpha=2-5$ ) followed by a shallow plateau $(\alpha=0-1)$ that finally breaks to the pre-Swift slope after a few hours. Flares with sharp rise and decay times are often superimposed to this global evolution.

Between the prompt emission and the initial decay phase, a hard to soft evolution is generally observed (e.g. [24]). Then, a soft to hard jump is often seen at the beginning of the plateau phase [24] which indicates the emergence of a new emission component. The spectral index remains approximately constant over the break between the plateau and the normal afterglow phase. This break therefore does not correspond to a spectral break (e.g. [11,25]). The flares share many common properties with the pulses of the prompt emission $[18,26,27,35]$ (general shape, brightness-hardness correlation, i.e. on the rise of the flare the spectrum is harder than during the decay) except that late flares last longer [28] (with $\Delta t / t \sim 0.1-0.3, \Delta t$ being the flare duration and $t$ its time of occurence).

The initial steep decay, plateau and flares components are not always present. Flares are observed in about $50 \%$ of the bursts. The plateau can be absent and the afterglow then follows a single power law already from the beginning of the XRT observations (the most extreme case being GRB 061007 [30] which maintained a constant slope $\alpha=1.6$ from 100 s to more than 10 days after trigger). Conversely, the plateau can be very flat and extended as in GRB 060729 [23] where it lasted nearly one day (Fig. 4).

\subsubsection{Implications for afterglow models}

The features of the early X-ray afterglow described above were quite unexpected and represent difficult challenges for the theoretical models.

- Initial steep decay: this phase might be the easiest to explain in the context of the standard model where the prompt emission comes from internal shocks taking place inside a relativistic outflow emerging from 

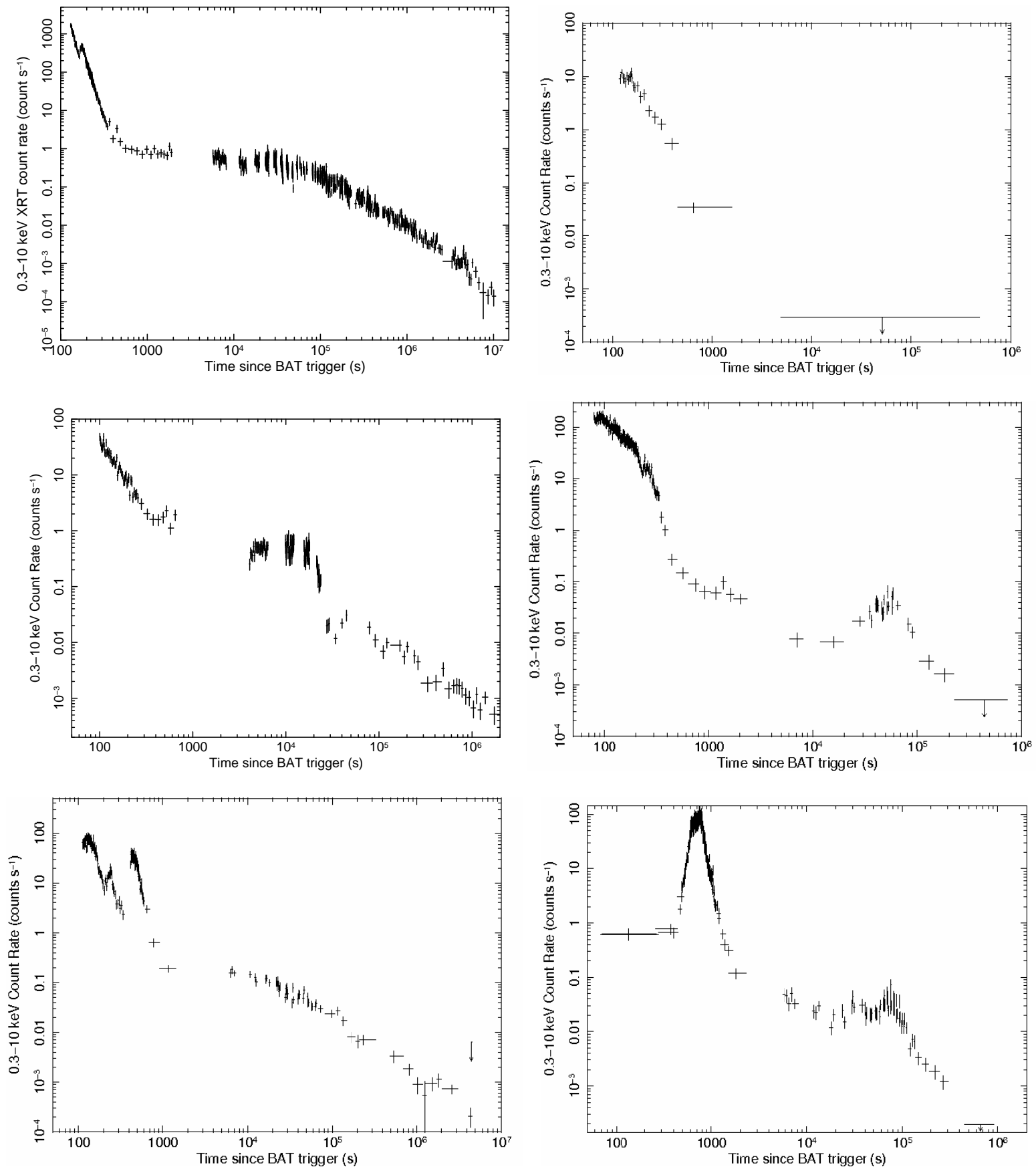

Figure 4. Examples of Swift-XRT light-curves: GRB 060729 (extended plateau; Top left); GRB 050421 (no afterglow; Top right); GRB 070110 (flat plateau ended by a steep drop; Middle left); GRB 050724 (short burst; Middle right); XRF 050822 (X-Ray Flash [29]; Bottom left); GRB 050502B (giant flare; Bottom right). The light-curves were obtained using the on-line lightcurve generator from [31]. 

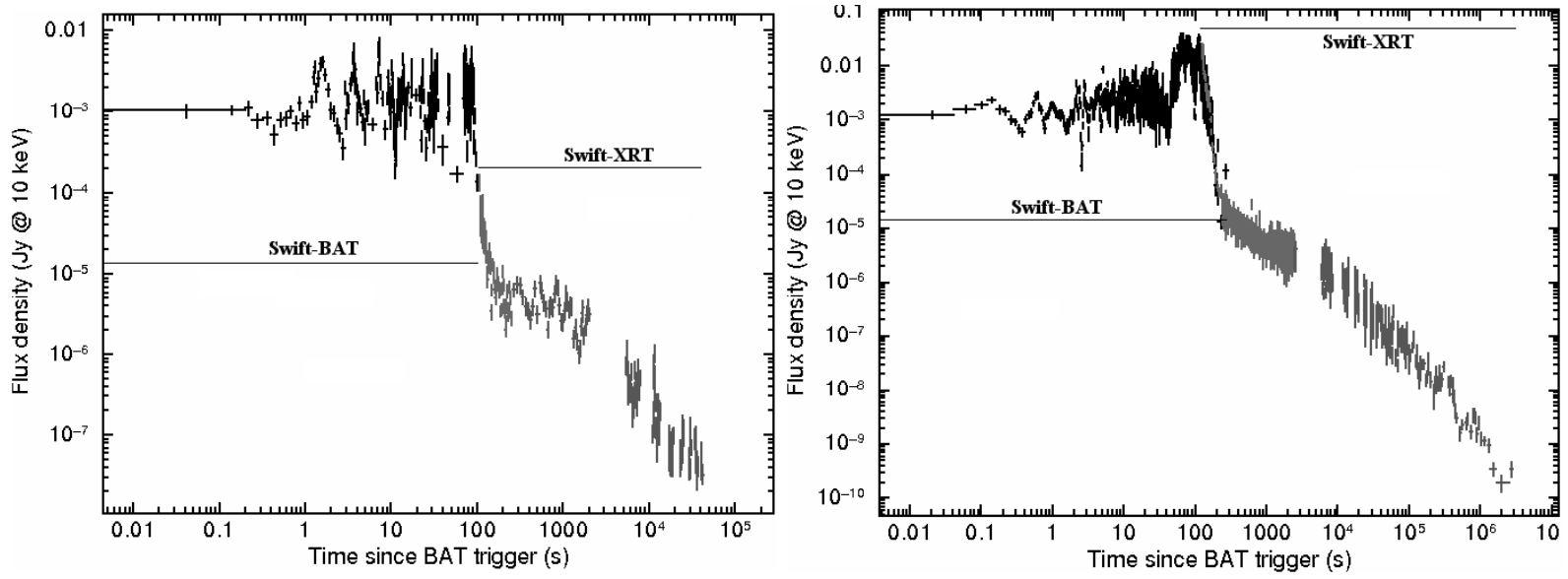

Figure 5. Examples of Swift combined BAT-XRT lightcurves showing the smooth connection between the prompt and steep decay of the early afterglow: GRB 080328 (Left); GRB 090618 (Right). The light-curves were obtained using the on-line lightcurve generator from [31].

the central engine. When internal shocks subside the last shocked shell flashes as a whole but, due to its curvature, the radiation emitted from regions away from the line of sight arrives to the observer with a delay, $\Delta t \sim R / 2 c \Gamma^{2}$ where $R$ is the shell radius and $\Gamma$ its Lorentz factor. It also benefits less from the "Doppler boost", which amplifies the flux in the direction of fluid motion. Consequently the flux received by the observer decreases. It can be shown that it follows a steep power law decay of the form $F_{\nu} \propto t^{2+\beta}$ where $\beta \sim 1-2$ is the spectral slope at $\nu$ [32]. This behavior is in global agreement with the data $[11,33,34,35]$. Figure 5 displays two examples of Swift combined BAT-XRT light-curves that show the smooth connection between the end of the prompt emission and the steep decay. As can be seen in Fig. 5, slopes steeper than 4 are sometimes observed that could be explained by a shift in the origin of time for the last pulses in the prompt light curve (i.e. taking the origin of time at the beginning of the pulse and not at the trigger).

In some cases, spectral evolution is observed during the initial steep decay with no corresponding change in the temporal slope even in XRT light-curves without X-ray flares superimposed (e.g. [36]). This spectral evolution may be due to other emission components coming from shock breakout or the rise of the afterglow, for instance.

Alternatives to internal shocks may explain the initial steep decay of the afterglow in the same way as long as the radius where the dissipation phase ends exceeds $10^{15} \mathrm{~cm}$. This could be for example the case for some reconnection models in a magnetized ejecta such as the "ICMART" model [37]. Conversely if the prompt emission comes from comptonization (multiple inverse Compton scatterings) near the photopheric radius $\left(10^{11}-10^{12} \mathrm{~cm}\right)[38,39]$ the delay $\Delta t$ induced by the curvature of the emitting shell will be much too short and the prompt phase will stop very abruptly. The observed initial decay of the afterglow should then result from an effective behavior of the central engine rather than from geometry. Another alternative to the curvature emission model invokes the breakout of the jet cocoon from the stellar envelope [40].

- Plateau phase: if the afterglow results from the forward shock propagating in the circumburst medium the most natural way to explain the plateau phase is to suppose that energy is added continuously to the shock so that the decline of the afterglow becomes temporarily shallower $[11,41]$. This can be done in two different ways: ( $i$ ) the central engine emits in a short time (the duration of the prompt phase) material with a distribution of Lorentz factors extending down to small values. As this ejecta is progressively decelerated the slower material is able to catch up and adds part of its energy to the forward shock; (ii) the central engine is active for a longer time (the duration of the plateau phase corrected for cosmic time dilation) and continuously emitting fast material that can also catch up and add energy to the shock.

Energy injection can momentarily reduce the afterglow decline, in agreement with observations. Two potential difficulties however arise from this assumption. First, the kinetic energy which is measured from multiwavelength fits of the afterglow now includes this late supply since the fits are performed after a few hours. Then, the energy which was available in the outflow during the prompt phase is reduced, which imposes an increased efficiency to the prompt mechanism in order to produce the same observed output in gamma-rays. In the case of bursts with an extended plateau this imposes an efficiency $E_{\gamma} / E_{\mathrm{K}}>50 \%$, out 
of the reach of most models invoked to explain the prompt emission. The second potential problem only concerns the second mode (ii) of energy injection as it supposes that the central engine remains active for up to one day to sustain the most extended plateaus. This might be possible $[44,45,46,47]$ but clearly represents a strong constraint on the central engine, especially for short bursts.

Alternatives to energy injection invoke: $(i)$ a delayed energy transfer from the forward shock to the electrons from the ISM that gives rise to a long and slow rise of the afterglow [48]; (ii) an off-beam jet so that when the jet decelerates the visible brightness surface enters the observer line of sight leading to the rise of the afterglow [49]; (iii) making the afterglow with a long-lived reverse shock that would result from an ejecta having a low Lorentz factor tail ([50,51], see Sect.3.3.1 below).

Some plateaus are even more puzzling as they end with a very steep decay, the best example being GRB 070110 where $F(t) \propto t^{-9}$ (Fig.4). As for flares (see below), this strong variability on a short timescale excludes a forward shock origin. It has therefore been proposed that these peculiar plateaus result from some kind of dissipation occurring at small radii, in a persistent outflow produced by an active magnetar (they are then called "internal plateaus" $[6,55])$.

- Flares: it appears very unlikely that flares are produced by the forward shock. Density clumps in the external medium being swept up by the shock only produce weak bumps with rise and decay times which are much too long [52]. A fast shell emitted by the central engine and adding its energy to the forward shock (refreshed shock) would lead to an increase of the underlying afterglow level after the flare which is not observed [53]. As a result, it is generally thought that flares originate from a late activity of the central engine $[11,44,45,46,47]$. This is supported by the fact that flares are similar to the pulses observed during the prompt phase except that late flares last longer, which is not the case for the prompt pulses. This imposes a very specific behavior to the central engine that will have to be explained (see [54] for one possibility).

\subsection{The afterglow at lower wavelengths}

\subsubsection{Optical}

During the pre-Swift era reasonable joint fits of the X-ray, optical and radio data have been obtained for many bursts and used to constraint the density of the external medium and the burst parameters (e.g. [14,15,16]). Due to the limited sensitivity of the X-ray instruments in BeppoSAX and HETE 2, the optical data was more complete than the X-rays which generally went below the detection limit after one day. The situation changed with the Swift XRT instrument which has been able to detect an afterglow in more than $90 \%$ of the bursts, now leaving many events with uncomplete optical coverage.

Pre-Swift optical observations seem to suggest the existence of two classes of optical afterlows: dark and bright ones $[20,56]$. It is worth noting that such a dichotomy is not observed in X-rays. Optical observations in the Swift era have confirmed the existence of these dark GRBs $[21, ?]$ (i.e. a class of GRBs with very faint optical afterglows compared to X-rays even when observing at early times). Recent works $[58,60,62]$ suggested that a fraction of these dark GRBs may be high- $z$ events while another fraction could be due to absorption in the vicinity of the GRB progenitor.

The multiwavelength behavior of the early afterglow has revealed a great complexity: in some bursts the X-ray and optical light curves match together (following the plateau phase and breaking to an identical slope at the end) while in others the optical light curve keeps the same power law slope beyond the end of the plateau in X-rays (e.g. $[58,59,63,64])$. These chromatic breaks are a challenge to models. In the standard forward shock scenario, they may require that the microphysical parameters vary with time in a very specific way (e.g. [65]) or that the X-rays and optical do not come from the same location or/and radiation process (e.g. [66]). Models have for example been proposed where the X-ray emission results from the up-scattered forward shock radiation [67].

A more radical point of view consists to explain the whole afterglow by a long-lived reverse shock [50,51], which supposes that the source has produced an ejecta with a low Lorentz factor tail and that the forward shock is radiatively inefficient. This can be the case if the shock dissipated energy remains in the baryonic component with almost no transfer to accelerate electrons or/and amplify the magnetic field in the shocked medium (i.e. very small $\epsilon_{e}$ or $/$ and $\epsilon_{B}$ ). If this condition is satisfied the reverse shock may represent an interesting alternative to the standard scenario, accounting for many of the observed peculiar temporal and spectral features.

It appears in conclusion that the diversity in the multiwavelength behavior of the early afterglow makes the modelling of this phase very difficult. It contrasts with the relative simplicity of the afterglow which was known in the pre-Swift era. The forward shock scenario which seemed able to explain the data after a few hours does not work (at least in its simplest version) at earlier times. It is still not clear if it can be adapted with some new 
ingredients - injection of energy, varying microphysics parameters - or if a true change of paradigm - afterglow made by the reverse shock, different origins for the X-ray and optical emissions - will be necessary.

\subsubsection{Radio}

Due to self-absorption at early times the radio follow-up of GRBs typically starts after a few days but can extend to several months and even years (for instance GRB 030329 [68]). At these late times it is expected that the forward shock becomes non-relativistic and that the jet expands laterally eventually reaching a quasi-spherical shape. In that stage the whole shocked material is directly accessible to observation and its energy content can be estimated. This so-called radio calorimetry has yielded typical values for the total energy release of a few $10^{51}$ erg $[69,70]$.

VLBI radio observations have been able to resolve the afterglow of some bursts and then used to follow the evolution of its apparent size as a function of time. Comparison to models provide constraints on the energy and opening angle of the jet as well as on the nature of the external medium (uniform density or stellar wind). For the few events for which such an analysis has been possible a (beaming corrected) value of the kinetic energy $E_{\mathrm{K}} \sim 10^{51} \mathrm{erg}$ of the outflow has been found, in agreement with the estimate from calorimetry [71,72].

A possible difficulty with the standard afterglow model arises at early times ( 1 - 10 days) where the observed radio temporal slopes are often very shallow and do not agree with (at least the simplest) theoretical predictions [73]. [73] discuss possible solutions to this problem and favor a scenario where the emission comes from a long-lived reverse shock propagating in material continuously outflowing from the central source.

\subsection{High energy long lasting emission}

Fermi LAT (Large Array Telescope [74]) observations have shown that the high energy emission (above 100 $\mathrm{MeV}$ ) typically starts after the emission in the $\mathrm{keV}$ - MeV range (as seen by the GBM - Gamma-ray Burst Monitor [75]) and lasts longer. It is not clear if the data correspond to the afterglow already from the beginning or if it should be explained by a superposition of prompt and afterglow components. Some hints of variability in the $\mathrm{GeV}$ range in possible coincidence with the $\mathrm{keV}-\mathrm{MeV}$ light curve may indicate a prompt origin for the early GeV emission [76] but then one has to explain why the subsequent afterglow component connect almost perfectly with the prompt phase. Indeed, in the few bursts for which LAT data cover both the prompt phase and early afterglow, no steep decay is observed at the end of the prompt phase (except maybe in GRB 090510 [77,78]) in contrast with what is seen in X-rays.

Moreover the radiative process at the origin of the high energy emission is not known. Some spectra can be well fitted by a broken power law (Band function [79]) from the keV to $\mathrm{GeV}$ range while others require an additional power law or a thermal component. Most models invoke synchrotron or/and Inverse Compton emission of shock accelerated electrons to explain the data, but hadronic models where the GeV photons and the additional power law come from hadronic processes have also been considered [80]. Only one burst (the short GRB 090510) has been simultaneously seen by Swift and the Fermi LAT starting from the trigger time. More such common detections can be expected in the future and should help to better constrain the radiation processes at work.

More details on the high energy emission of GRBs will be found in [81] (this issue).

\section{The afterglow of short GRBs and X-ray flashes}

\subsection{The afterglow of short GRBs}

Short bursts tend to be harder than long bursts (e.g. [82]) and emit less photons which made their detection and localization more difficult. The first afterglows of short bursts were found only in 2005 (one by HETE-2 GRB 050709 [83] - and all the others by Swift $[84,85,86]$ and references therein).

The nature of the host galaxies, the location of the GRBs within the host galaxies and the absence of a supernova imprint in the visible light curve (even when the host is located at a redshift below 0.5) suggests that the progenitors of short bursts are different from those of long bursts. Several short bursts are clearly associated to elliptical galaxies while others with accurate positions appear to have no coincident host, which clearly excludes progenitors belonging to the young population and favors merger scenarios involving compact objects - two 
neutron stars or a neutron star-black hole binary $[87,88,89]$. Short GRBs are located at lower redshift $(\langle z\rangle \sim 0.4)$ than long ones but recently, a population of high- $z(z>1)$ short GRBs may have been detected (see [90]).

Afterglows of short bursts fall in two classes: about $40 \%$ of the events have no detectable afterglow after about $1000 \mathrm{~s}$ while the other $60 \%$ have long lasting afterglows comparable to those of long GRBs. Short GRBs appear as less energetic events when compared to long GRBs with a gamma-ray jet corrected energy around $10^{49} \mathrm{erg}$ (against $10^{51} \mathrm{erg}$ for long GRBs (e.g. [91] for instance). If indeed short bursts result from the merging of two compact objects the kick received when the neutron star or black hole components formed in supernova explosions allows the system to reach the low density outskirts of the host galaxy (or even to leave the galaxy) before coalescence occurs. This can naturally explain why a fraction of the afterglows are so dim or have no coincident host. It is interesting to note that in the case of a few long GRBs (such as GRB 050421 [92,93] - see Fig.4) no afterglow or a dim X-ray afterglow was detected even if Swift started promptly observing the GRB position after the Swift-BAT trigger, which might suggest that these events are "naked GRBs" with ISM density as low as that observed for some short GRBs [86].

Some flaring activity has also been seen in short bursts up to several hundreds seconds after trigger such as in GRB 061210 or GRB 100117A. In addition to flares a wide bump was also present at much later times (between $5 \times 10^{4}$ and $10^{5} \mathrm{~s}$ ) in GRB 050724 (see Fig.4). If flares result from a persistent activity of the central engine this would imply that it can still operate even in bursts where the prompt phase lasted less than one second (for instance $200 \mathrm{~ms}$ in GRB 061210 [94]).

The multi-wavelength observations of afterglows of short GRBs also led to constrain the opening angle of the jets that appear to be less collimated than in long GRBs [91,95].

\subsection{X-ray flashes}

X-ray flashes (XRFs) share many common properties with GRBs except that their spectrum peaks in the Xray range (from a few $\mathrm{keV}$ to a few tens of $\mathrm{keV}$ ). Observations of XRF afterglows have helped to answer several questions regarding their nature and origin. First, they confirmed that XRFs are, as GRBs, located at cosmological distances. The first redshift was measured for XRF 020903 at $z=0.25$ [96] but larger values (up to $z=2.65$ for XRF 030429 [97]) were later obtained.

Another issue was to decide if XRFs were intrinsically soft or if their low $E_{\mathrm{p}}$ (the peak energy of the $\nu F_{\nu}$ spectrum) resulted from special observational circumstances. A first possibility, a large redshift, was clearly excluded already with XRF 020903 which had an $E_{\mathrm{p}}$ of a few $\mathrm{keV}$ at $z=0.25$. It was also suggested that XRFs were soft events because they were seen off-axis with a reduced Lorentz boost in the direction of the observer. Such a geometry delays the rise of the afterglow until the angle $\theta_{j}+1 / \Gamma$, where $\theta_{j}$ is the physical opening angle of the jet and $1 / \Gamma$ the relativistic beaming angle (which is increasing as a result of deceleration) can encompass the line of sight. Afterglow observations can then be used to test the off-axis scenario but the available data has not yet provided a fully clear conclusion. XRF 030723 [98] and XRF 080330 [99] seem to be consistent with an off-axis jet while XRF 020903, 050215B [100], 050406 [101], 050416A [102] (see also [103]) and 050822 [104] appear to have been intrinsically soft. [105] also suggested that the softness of XRFs is due to a wider jet opening angle compared to classical GRBs. [102,104] showed in the case of XRF 050822 and 050416A that the jet opening angle is likely to be more than $20^{\circ}$, which is much larger than the average value $\theta_{j} \sim 5^{\circ}$ derived for GRBs by [106].

\section{Current issues and problems}

In addition to the questions raised by the early afterglow or the high energy emission we list below a few additional problems faced by standard afterglow modelling:

\subsection{The jet break problem}

As the ejecta is decelerated by the external medium relativistic beaming of the emitted radiation becomes less effective and $1 / \Gamma$ becomes eventually larger than the physical opening angle of the jet $\theta_{j}[107,108]$. This leads to an achromatic break in the light curve (since it is a purely geometrical effect - see Fig. 3 ). The time $t_{j}$ of this "jet break" can be used to estimate $\theta_{j}$ and therefore the true energy release of the burst, corrected for beaming $[106,109]$. In the pre-Swift era, candidate jet breaks were identified at typical times $t_{j} \sim$ one day and yielded 
values of the energy in gamma-rays $E_{\gamma}$ of a few $10^{50} \mathrm{erg}$, suggesting the existence of a standard energy reservoir $[106]$.

The situation became less clear in the Swift era as it is now possible to follow some X-ray afterglows for several weeks and even months. In many cases no break is observed (e.g. [25,110], see however [111]); which seems to indicate beaming angles and emitted energies larger than previously expected. In a study of 5 bright bursts [112] were able to identify late jet breaks at about 10 days. The corresponding beaming angles were all larger than $5^{\circ}$ (with $\theta_{j}>20^{\circ}$ in one case). The true energy in gamma-rays then reaches $10^{52} \mathrm{erg}$ for these bright bursts, much above what was believed to be the standard value. One possible way to avoid increasing too much the energy budget of the central engine would be to suppose that the radiation is not isotropic in the comoving frame of the emitting material [113]. Then, it would be beamed in an angle smaller than $1 / \Gamma$ in the observer frame and, for a given physical opening angle of the jet, the jet break will be delayed.

It should finally be mentioned that the new situation revealed by Swift casts doubts about the early jet breaks from the pre-Swift era. It cannot be excluded that at least some of them corresponded to the end of the plateau phase and were misinterpreted as "jet breaks".

\subsection{Nature of the circum-burst medium}

It has been mentioned in Section 2 that, in the framework of the standard model, multi-wavelength fits of afterglows seem to favor a low density, uniform external medium, even for long bursts (e.g. [11,114,115]). This is somehow unexpected since long bursts are supposed to be produced by some extreme kind of type Ib/c supernovae [116]. The progenitors of type Ib/c are Wolf-Rayet stars which normally have strong winds. One possible solution could be that in most cases the radius of the wind-termination shock is relatively small so that the jet deceleration mostly happens after this radius. The requirement of the collapsar model that the progenitor star keeps a large angular momentum up to its collapse indeed implies a lower mass loss [117], which is consistent with the possibility of a small termination shock radius.

It nevertheless remains surprising that more weight is often given in the literature to the results of a modelling process that rely on many uncertain parameters and assumptions (such as taking constant values of $\epsilon_{e}$ and $\epsilon_{B}$ ) than to standard astronomical knowledge...

\subsection{Microphysics}

We just indicated that most of the modelling work is performed by adopting fixed values of the microphysics parameters. This is the most natural zeroth-order assumption but it is not necessarily true. The problem is that, unfortunately, shock physics has not reached the status where reliable predictions can be made on how these parameters should vary with the shock strength or density of the external medium. Allowing $\epsilon_{e}$ and $\epsilon_{B}$ to vary obviously adds considerable freedom to the modelling process but also makes it very uncertain with limited predictive power. For example the often used "closure relations" [11] that link the temporal and spectral indices in the different regimes of afterglow evolution rely on the assumption that the microphysics parameters stay constant. The preference for a uniform external medium also results from the same assumption. If the microphysics parameters can vary, wind models can be more easily accomodated.

Also, the distribution of the power-law index $p$ of the electron population appears far from universal [11,118], contrary to what is expected from theoretical calculations and simulations of particle acceleration in relativistic shocks $(p=2.2-2.3)$. This is puzzling but could also be a consequence of inappropriate assumptions regarding $\epsilon_{e}$ and $\epsilon_{B}$.

\subsection{Consistency of the global model}

It should finally be reminded that any new ingredients added to one part of the model should be consistent with the rest of it! In that respect some of the solutions proposed to solve problems in afterglow physics have important consequences for the prompt phase (and vice-versa).

- Initial steep decay: it is tempting to interpret the initial steep decay at the end of the prompt phase as the high latitude emission of the last flaring shell. This supposes that the delay $\Delta t=R / 2 c \Gamma^{2}$ ( $R$ being the radius of the shell and $\Gamma$ its Lorentz factor) is not much smaller that the duration of the prompt phase. This 
condition is naturally satisfied by internal shocks but not by a dissipative photosphere and by most magnetic reconnection models where $\Delta t$ is too short.

- Energy injection is generally invoked to explain the plateau phase of the early X-ray afterglow. We already explained why such a scenario implies a very high efficiency for the prompt phase which practically excludes internal shocks and challenges most other models. Moreover the propagation of the reverse shock through the material adding its energy to the forward shock will add a new contribution to the early afterglow which has never been properly estimated.

- Models where the afterglow is made by the reverse shock obviously imply that a reverse shock is present which is not the case in a magnetized ejecta where the magnetization $\sigma=E_{\mathrm{mag}} / E_{\mathrm{K}}>1$. Therefore such models either require that the outflow emerging from the central engine is not magnetized or that most of the magnetic energy has already been converted to kinetic energy when the reverse shock develops.

\section{Conclusion}

Following the discovery of the afterglows by Beppo-SAX, it was generally believed that their physics would be much simpler than that of the prompt phase of GRBs. The basic framework involved a relativistic generalization of the classical Sedov problem and part of the theory (the Blandford-McKee solution) even preexisted the 1997 discovery. The situation became more confuse in the Swift era when the early afterglow was first observed. The standard forward shock model (at least in its basic version) appeared unable to account for the new phenomenology (the plateau phase and the flares) revealed by Swift.

Solutions supposing a long term activity of the central engine that have been proposed to explain both the plateau and the flares are not free of pitfalls: they increase the required efficiency of the prompt phase to levels that might be incompatible with most models, especially internal shocks. Flares impose a specific, intermittent behavior to the source that is different from the more continuous and regular activity needed to sustain the plateau.

Proposed alternatives to the standard model rely on new assumptions that will have to be confirmed by further confrontation to the data. For instance, the role played by the reverse shock remains uncertain. The reverse shock may simply not exist if the flow is still highly magnetized when it starts to be decelerated. It will be short lived if the ejecta only contains material of high Lorentz factor and then might be responsible for the early optical flashes that have been seen in a few bursts. Finally if the ejecta has a low Lorentz factor tail, the reverse shock will be long-lived and may possibly contribute to the afterglow.

Fermi LAT observations also are not easy to interpret. The high energy emission extends beyond the end of the prompt phase and should somehow be linked to the afterglow, at least at late times. However it is not clear if it simply corresponds to the high energy tail of the usual afterglow or if it involves a new, possibly hadronic component.

It finally appears that the results from the Swift and Fermi satellites have not contributed to clarify our understanding of afterglow physics. Instead they have added new puzzles and raised new questions! This may appear as a frustrating and pessimistic conclusion but one should bear in mind the difference between progress in understanding and progress in knowledge. In the past fifteen years we have made tremendous progress in our knowledge of GRBs. This is especially true for afterglows which simply "did not exist" before 1997. We have now collected a huge amount of multiwavelength data starting from one minute after the trigger. Future missions like SVOM (to be launched in 2016) will continue this quest. SVOM will not only obtain GRB key parameters such as $E_{\mathrm{p}}$ and the redshift, but will also provide well-sampled multi-wavelength light-curves from early to late times. In addition to a fast slew capability of the satellite and sensitive multi-wavelength NFIs providing accurate positions, this imposes to build a strong synergy between space and ground via an international network of robotic telescopes. This will ensure that large facilities such as the VLT are able to perform observations as quickly as possible.

One can then hope that increasing our knowledge of GRBs will ultimately lead to more understanding...

\section{References}

[1] E. Costa et al., IAU Circular (1997) 6572

[2] L. Scarsi et al., AdSpR (1993) 131, 369 
[3] D.Q. Lamb et al., NewAR (2004) 48, 423

[4] N. Gehrels et al., ApJ (2004) 611, 1005

[5] P. Petitjean, GRBs as probes of the distant universe, C. R. Physique 12 (2011), (this issue)

[6] B. Zhang, Open questions in GRB physics, C. R. Physique 12 (2011), (this issue)

[7] P. Mészáros \& M.J. Rees, ApJ (1997) 476, 232

[8] R.D. Blandford \& C.F. Mckee, Phys. of Fluids (1976) 19, 1130

[9] M. Lemoine \& G. Pelletier, Shock acceleration in Gamma-Ray Bursts, C. R. Physique 12 (2011), (this issue)

[10] R. Sari, T. Piran \& R. Narayan, ApJ (1998) 497, L17

[11] B. Zhang, et al., ApJ (2006) 655, 989

[12] Z.-Y. Li \& R.A. Chevalier, ApJ (2001) 551, 940

[13] A. Panaitescu \& P. Kumar, ApJ (2000) 543, 66

[14] A. Panaitescu \& P. Kumar, MNRAS (2004a) 353, 511

[15] A. Panaitescu \& P. Kumar, MNRAS (2004b) 350, 213

[16] A. Panaitescu \& P. Kumar, ApJ (2001) 554, 667

[17] S.D. Barthelmy et al., Space Science Reviews (2005) 120, 143

[18] D.N. Burrows et al., Space Science Reviews (2005) 120, 165

[19] P.W.A. Roming et al., Space Science Reviews (2005) 120, 95

[20] E. Rol et al., ApJ (2005) 624, 868

[21] P.W.A. Roming et al., ApJ (2006) 652, 1416

[22] V. Mangano et al., Il NCim B (2006) 121, 1297

[23] D. Grupe et al., ApJ (2007) 662, 443

[24] P.T. O'Brien et al., ApJ (2006) 647, 1213

[25] R. Willingale et al., ApJ (2007) 662, 1093

[26] G. Chincarini et al., ApJ (2007) 671, 1903

[27] A.D. Falcone et al., ApJ (2007) 671, 1921

[28] P. Curran et al., A\&A (2008) 487, 533

[29] J.-L. Atteia \& M. Boër, Observing the prompt emission of GRBs, C. R. Physique 12 (2011), (this issue)

[30] P. Schady et al., MNRAS (2006) 380, 1041

[31] P.A.E. Evans et al., MNRAS (2009) 397, 1177

[32] P. Kumar \& A. Panaitescu, ApJ (2000) 541 , L51

[33] G. Tagliaferri et al., Nature (2005) 436, 985

[34] M. Goad et al., A\&A (2006) 449, 89

[35] E.W. Liang et al., ApJ (2006) 646, 351

[36] A. Moretti et al., A\&A (2008) 478, 409

[37] B. Zhang \& H. Yan, ApJ (2011) 726, 90

[38] M.J. Rees \& P. Mészáros, ApJ (2005) 628, 847

[39] A. Beloborodov, MNRAS (2010) 407, 1033

[40] A. Pe'er, P. Mészáros \& M.J. Rees, ApJ (2006) 652, 482

[41] J. Nousek et al., ApJ (2006) 642, 389

[42] E. Troja. et al., ApJ (2007) 665, 599

[43] S. Campana et al., Nature (2006) 442, 1008

[44] A.R. King et al., ApJ (2005) 630, 113

[45] R. Perna, P.J. Armitage \& B. Zhang, ApJ (2006) 636, 29 
[46] D. Proga \& B. Zhang, MNRAS (2006) 370, 61

[47] S. Rosswog, MNRAS (2007), 376, 48

[48] S. Kobayashi \& B. Zhang, ApJ (2007) 655, 973

[49] D. Eichler \& J. Granot, ApJ (2006) 641, 5

[50] F. Genet, F. Daigne \& R. Mochkovitch, MNRAS (2007) 381, 732

[51] Z.L. Uhm \& A. Beloborodov, ApJ (2007) 665, L93

[52] E. Nakar \& J. Granot MNRAS (2007) 380, 1744

[53] J. Granot, E. Nakar \& T. Piran Nature (2003) 426, 138

[54] D. Lazzati et al., MNRAS (2011) 411, L16

[55] N. Lyons et al., MNRAS (2009) 402, 705

[56] P. Jakobsson et al., ApJ (2004) 617, 21

[57] D.W. Fox et al., ApJ (2003) 586, 5

[58] A. Melandri et al., ApJ (2008) 686, 1209

[59] A. Panaitescu \& W.T. Vestrand, MNRAS (2008) 387, 497

[60] D.A. Perley et al., AJ (2009) 138, 1690

[61] S.B. Cenko et al., ApJ (2009) 693, 1484

[62] J. Greiner et al., A\&A (2011) 526, 30

[63] P.W.A. Roming et al, ApJ (2009) 690, 163

[64] D.A. Kann et al., ApJ (2010) 720, 1513

[65] A. Panaitescu et al., MNRAS (2006) 369, 2059

[66] G. Ghisellini et al., MNRAS (2009) 393, 253

[67] A. Panaitescu MNRAS (2008) 383, 1143

[68] A.J. Van der Horst et al., NCim B (2006) 121, 1605

[69] D.A. Frail, E. Waxman \& S.R. Kulkarni, ApJ (2000) 537, 191

[70] D.A. Frail et al., ApJ (2005) 619, 994

[71] Y. Oren, E. Nakar \& T. Piran, MNRAS (2004) 353, L35

[72] Y.M. Pihlström et al., ApJ (2007) 664, 411

[73] A. Panaitescu \& P. Kumar, MNRAS (2004) 350, 213

[74] W.B. Atwood et al., ApJ (2009) 697, 1071

[75] P.N. Bhat et al., in Gamma-Ray Bursts: Sixth Huntsville Symposium. AIP Conference Proceedings (2009) 1133, 34

[76] B.B. Zhang et al., arXiv:1009.3338v2 (2010)

[77] M. Ackermann et al., ApJ (2010) 716, 1178

[78] G. Ghirlanda, G. Ghisellini \& L. Nava, A\&A 510, L7

[79] D. Band et al., ApJ (2003) 413, 281

[80] K. Asano, S. Guiriec \& P. Mészáros, ApJ (2009) 705, L191

[81] F. Piron \& V. Connaughton, The Fermi view of gamma-ray bursts, C. R. Physique 12 (2011), (this issue)

[82] R.D. Preece et al., ApJS (2000) 126, 19

[83] D.B. Fox et al., Nature (2005) 437, 845

[84] N. Gehrels et al., Nature (2005) 437, 851

[85] S. Barthelmy et al., Nature (2005) 438, 994

[86] B. Zhang, Chinese Journal of Astronomy \& Astrophysics (2007) 7, 1

[87] B. Paczyński, ApJ (1986) 308, L43

[88] D. Eichler et al., Nature (1989) 340, 126 
[89] S. Rosswog et al., MNRAS (2003) 334,481

[90] E. Berger et al., ApJ (2007) 664, 1000

[91] D.N. Burrows et al., ApJ (2006) 653, 468

[92] O. Godet et al., A\&A (2006) 452, 819

[93] L. Vetere et al., in Gamma-ray Bursts: Sixth Huntsville Symposium. AIP Conference Proceedings (2009) 1133, 199

[94] P.A. Cumin et al., A\&A (2007) 467, 1049

[95] D. Watson et al., A\&A (2004) 454, L123

[96] A.M. Soderberg et al., ApJ (2004) 606, 994

[97] P. Jakobsson et al., ApJ (2004) 427, 785

[98] N.R. Butler et al., ApJ (2005) 621, 884

[99] C. Guidorzi et al., A\&A (2009) 499, 439

[100] A.J. Levan et al., ApJ (2006) 648, 1132

[101] P. Romano et al., A\&A (2006) 450, 59

[102] V. Mangano et al., ApJ (2007) 654, 403

[103] S.T. Holland et al., AJ (2007) 133, 122

[104] O. Godet et al., A\&A (2007) 471, 385

[105] D.Q. Lamb, T.Q. Donaghy \& C. Graziani, NCim C (2005) 28, 365

[106] D.A. Frail et al., ApJ (2001) 562, L55

[107] J.E. Rhoads, ApJ (1999) 525, 737

[108] J.E. Rhoads, ApJ (1997) 487, L1

[109] E. Liang \& B. Zhang, ApJ (2005) 633, 611

[110] E. Liang et al., ApJ (2009) 707, 328

[111] J.L. Racusin et al., ApJ (2009) 698, 43

[112] S.B. Cenko et al., ApJ (2010) 711, 641

[113] A.M Beloborodov et al., MNRAS (2010) 410, 2422

[114] A. Panaitescu \& P. Kumar, ApJ (2001) 560, L49

[115] S. Schulze et al., A\&A (2011) 526, 23

[116] S.E. Woosley, ApJ (1993) 405, 273

[117] N. Langer, A.-J. van Marle \& S.-C. Yoon, New Astronomy Reviews (2010) 54, 206

[118] R. Shen, P. Kumar \& E.L. Robinson, MNRAS (2006) 371, 1441 